are transported to the surface and give rise to sunspots.

We have, in particular, considered an axially symmetric model for the Sun. In a first approximation we neglect viscosity and Coriolis forces, and consider the case of azimuthal motion of the fluid particle along a magnetic surface (i.e., the surface of revolution generated by rotation of a line of force about the axis of symmetry). The equations of the problem are then rigorously linear. We have solved them by a variational technique to determine the fundamental periods. We have taken the lower boundary of the convective layer to be a nodal surface for the velocity. In the present approximation, motions in different magnetic surfaces are independent. With basic fields of the order of IOO-IOOO gauss in the equatorial plane, the periods obtained are of the order of Io years and consistent, within the limits of our knowledge, with the 22 -year value of the spot cycle. The maximum relative amplification of the field occurs at the nodal surface.

We are proceeding with the next approximation where inter alia coupling between different magnetic surfaces is taken into account.

The research reported in this paper has been supported by the Geophysics Research Directorate, Air Force Cambridge Research Center, under a contract with Harvard University.

Harvard College Observatory, Cambridge, Mass.

\section{Mestel, L. On the decay of the magnetic field in a proto-star.}

Rapid compression and cooling of a normal interstellar region is supposed to have formed a cloud of $\mathrm{IO}^{3}$ solar masses, about a parsec in radius, at a temperature of $\mathrm{IO}^{2}{ }^{\circ} \mathrm{K}$, and with a magnetic field of about $\mathrm{IO}^{-4}$ gauss. The magnetic, thermal and gravitational energies are then all of comparable magnitude. Sufficient dust particles are assumed present to absorb all the galactic starlight falling on the surface; the cloud as a whole then loses energy so rapidly that it falls freely under its own gravitation, remaining roughly isothermal. If the cloud is ultimately to fragment into stars of low magnetic energy, it must contract freely across the lines of force.

In the absence of external radiation, the ionelectron density begins to decay rapidly, chiefly through attachment to the dust particles; but it can be shown that the electron density cannot become low enough for the time of magnetic decay (through Joule heating) to approach the time of free-fall. However, Dr. Spitzer has pointed out that as the plasma density decreases, the frictional coupling between it and the neutral gas becomes small compared with the magnetic force, so that for moderately low plasma density the magnetic field effectively exerts no action on the neutral gas. Thus the initially distorted lines of force snap back, carrying with them the remains of the plasma, and the field inside the cloud returns to roughly the same value as in the surrounding medium. The cloud can then fragment into proto-stars of low magnetic energy. Dragging of the lines of force by the contracting cloud is resumed when the internal stellar temperatures exceed $\mathrm{IO}^{3}{ }^{\circ} \mathrm{K}$, so that free electrons are produced. The field in the ultimate main sequence condensation is of order $\mathrm{IO}^{2}$ or $\mathrm{ro}^{3}$ gauss.

Princeton University Observatory,

Princeton, N. J.

\section{Mikesell, Alfred H. Stellar scintillation and} meteorological phenomena.

Amplitudes of scintillation were available on a hundred nights spread over four years. They have been compared with the Weather Bureau radiosonde data concerning the lower $30 \mathrm{~km}$ of the atmosphere.

No aspect of the distribution of scintillation harmonics could be fully correlated with any specific feature of the wind and temperature distributions. Statistically, however, scintillation amplitude correlates with wind-speed observed at any level in the region eight to sixteen kilometers high. The best correlation is with maximum wind-speed, taken irrespective of height. The maximum wind-speed does not appear at sharply defined layers. It is rather the fastest point of a broad distribution of unidirectional winds which generally extend fairly uniformly from the low speeds near the surface of the earth to the low speeds found in the stratosphere.

There is poor correlation of scintillation, or of maximum wind-speed, with the heights of the tropopause, other temperature inversions, or temperature minima.

These data suggest a hypothesis whereby scintillation develops because of a large depth of isotropically turbulent atmosphere, rather than because of a few thin layers of special disturbance.

U. S. Naval Observatory, 